\title{
ThC1 INTEGRATED OPTICAL TRANSMITTERS AND RECEIVERS USING MULTI-SEGMENT LASER PROCESSES
}

\author{
J. G. Wasserbauer, D.J. Derickson, K. Giboney, R. J. Helkey, J. R. Karin, A. Mar, and J. E. Bowers \\ University of California, ECE Department, Santa Barbara, Ca. 93106
}

Research on integrated optical devices has often focussed on complex structures and complicated processing techniques $[1,2]$. However, a variety of integrated optoelectronic devices can be fabricated from a simple semiconductor laser diode waveguide process. Several important functional components can be obtained by splitting the top contact in a laser process into several segments allowing for non-uniform pumping. When forward biased, the device has gain allowing for amplification, direct current modulation of the gain, and modulation of the index of refraction. When reverse biased, the segment functions as a p-i-n photodetector, saturable absorber, and electro-absorption modulator. With these simple functions, many useful integrated optoelectronic devices can be formed. Figures 1a and $1 \mathrm{~b}$ show example transmitter and receiver devices which have been fabricated with our process.

Figure la shows a three-segment monolithic cavity mode-locked semiconductor laser [3] with a waveguide saturable absorber, gain modulation segment, and gain segment. Devices with repetition rates as low as $5.5 \mathrm{GHz}$ (7mm device length) and as high as $80 \mathrm{GHz}(0.5 \mathrm{~mm}$ device length) have been fabricated. Figure 2 shows an autocorrelation trace for a $1.55 \mu \mathrm{m}$ wavelength monolithic cavity mode-locked laser operating at a repetition rate of $21 \mathrm{GHz}$. Device structures similar to that of Figure la have been used for a 3-section gain-switched laser in which two saturable absorbers near the laser facets are used to obtain shorter pulsewidths than are possible with single-section gain-switched lasers. The device of Figure 1a can also be used as a two-segment superluminescent LED. A short reverse-biased segment acts as an optical termination to absorb spontaneous emission in one direction with a power reflection coefficient below $10^{-4}$. The reverse-biased optical termination segment also serves to monitor average power from the superluminescent LED.

Figure 1b shows a 3 segment pre-amplified photodetector in which one segment is used as an optical amplifier, the second segment is used as a switch to turn the signal to the detector on or off, and the third segment is the p-i-n photodetector. Figure 3 shows the impulse response of the waveguide photodetector with a mode-locked semiconductor laser as the pulse source. The full width at half of maximum is $33 \mathrm{ps}$ limited by the capacitance of the photodetector. The switching segment can be turned on and off in $200 \mathrm{ps}$ using a step-recovery diode drive signal. The switching segment can also be used as a saturable absorber in pulse amplification applications. The entire spontaneous emission output from the amplifier does not reach the photodetector between optical pulses. Pump-probe measurements of the saturable absorber section were made to measure the absorption recovery time constant. The saturable absorber can recover to the highly attenuating state in less than $10 \mathrm{ps}$ after the passage of the optical pulse through the absorber dem.unstrating that the saturable absorber can be effective in reducing the spontaneous emission reaching the photodetector.

An outline of a low capacitance multi-segment laser process is shown in Figure 4. A silicon nitride layer is deposited and patterned to outline the waveguide. The silicon nitride is used as an etch mask to define the waveguide for wet or dry chemical etching. Next, a polyimide layer is deposited and cured. In order to planarize the polyimide layer, a thick layer of photoresist is spun over the entire wafer. Both the polyimide and the photoresist layers are then etched in an oxygen plasma until the top of the mesa slightly protrudes through the polyimide layer. The silicon nitride layer is then removed and the wafer is ready for metallization. The multi-segment p-metal is evaporated, lifted off and annealed. The metal contacts are used as a 3-way self-aligned mask to 1) etch the contact layer between electrodes, 2) etch cleave marks for precise positioning of facet cleaves, and 3) remove the excess polyimide in order to promote good facet cleaving. Finally, the wafer is thinned, a backside metal is deposited and the devices are cleaved.

[1] U. Koren, Indium Phosphide and Related Materials Conference, April, 1990. Paper TuC.1.

[2] T.L. Koch, U. Koren, R.P. Gnall, F.S. Choa, F. Hernandez-Gil, C.A. Burrus, M.G. Young, M. Oron, and B.I Miller, Indium Phosphide and Related Materials Conference, April, 1990. Paper TuC.2.

[3] D. J. Derickson et al., 1992 Optical Fiber Conference, Paper ThB3, San Jose, Ca 


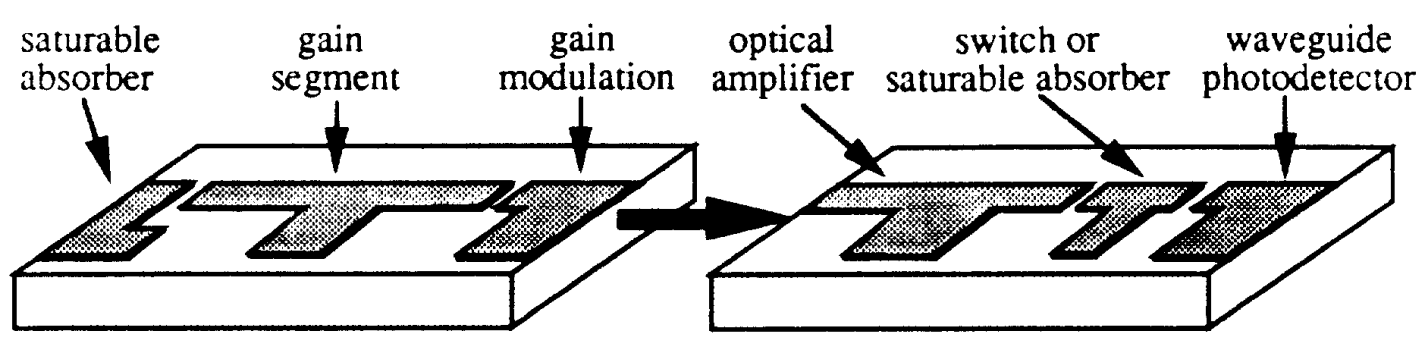

Fig. 1a. 3-section monolithic

Fig.1b: 3-section preamplified photodetector cavity mode-locked laser

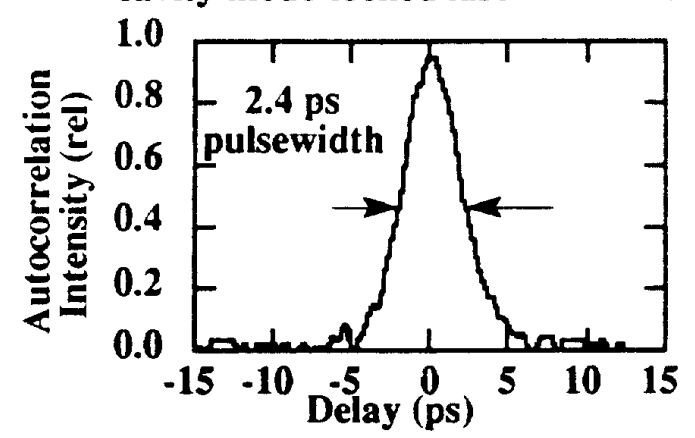
with switching segment and saturable absorber

Fig. 2. Mode-locked laser autocorrelation

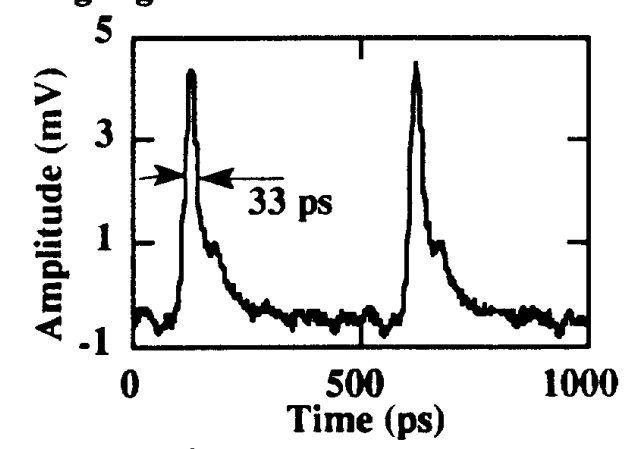

Fig. 3. Waveguide photodetector response
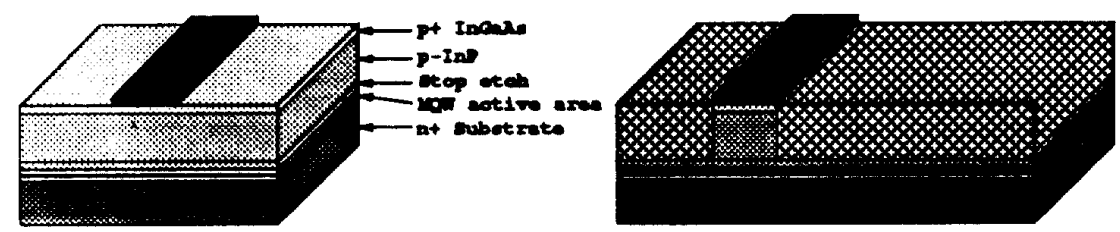

1) Nitride deposition

2) Waveguide pattern

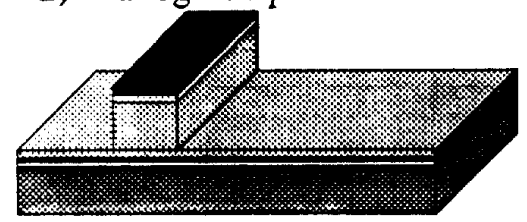

3) Wet etch

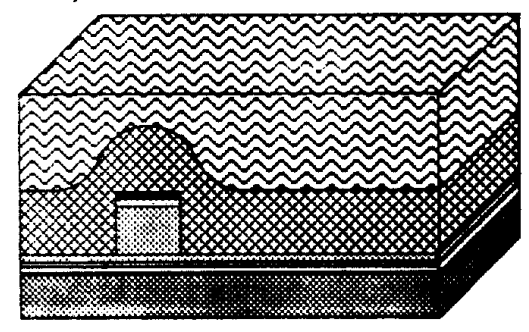

4) Polyimide deposition/cure

5) Photoresist spin

6) Planarizing $\mathrm{O}_{2}$ plasma etch

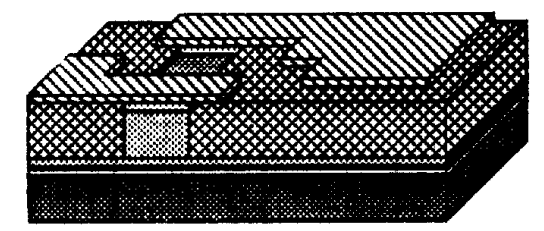

7) Liftoff contact metal

8) Contact separation etch

Fig. 4 . Low capacitance multi-section integration process 